Article

\title{
Two-Layer Erbium-Doped Air-Core Circular Photonic Crystal Fiber Amplifier for Orbital Angular Momentum Mode Division Multiplexing System
}

\author{
Hu Zhang ${ }^{1,2, *}$, Di Han ${ }^{1}$, Lixia Xi ${ }^{1, *}$, Zhuo Zhang ${ }^{3}$, Xiaoguang Zhang ${ }^{1}$, Hui Li ${ }^{1}$ and \\ Wenbo Zhang ${ }^{1,4}$
}

1 State Key Laboratory of Information Photonics and Optical Communications, Beijing University of Posts and Telecommunications, Beijing 100876, China; hd2016@bupt.edu.cn (D.H.); xgzhang@bupt.edu.cn (X.Z.); lihui1206@bupt.edu.cn (H.L.); zhangwb@bupt.edu.cn (W.Z.)

2 School of Ethnic Minority Education, Beijing University of Posts and Telecommunications, Beijing 100876, China

3 Institute of Electronics, Chinese Academy of Sciences, Beijing 100190, China; zhangzhuo@mail.ie.ac.cn

4 School of Sciences, Beijing University of Posts and Telecommunications, Beijing 100876, China

* Correspondence: zhh309@bupt.edu.cn (H.Z.); xilixia@bupt.edu.cn (L.X.); Tel.: +86-010-6119-8077 (H.Z.); +86-010-6119-8077 (L.X.)

Received: 25 February 2019; Accepted: 12 March 2019; Published: 15 March 2019

\begin{abstract}
Orbital angular momentum (OAM) mode-division multiplexing (MDM) has recently been under intense investigations as a new way to increase the capacity of fiber communication. In this paper, a two-layer Erbium-doped fiber amplifier (EDFA) for an OAM multiplexing system is proposed. The amplifier is based on the circular photonic crystal fiber (C-PCF), which can maintain a stable transmission for 14 OAM modes by a large index difference between the fiber core and the cladding. Further, the two-layer doped region can balance the amplification performance of different modes. The relationship between the performance and the parameters of the amplifier is analyzed numerically to optimize the amplifier design. The optimized amplifier can amplify 18 modes (14 OAM modes) simultaneously over the C-band with a differential mode gain (DMG) lower than 0.1 $\mathrm{dB}$ while keeping the modal gain over $23 \mathrm{~dB}$ and noise figure below $4 \mathrm{~dB}$. Finally, the fabrication tolerance and feasibility are discussed. The result shows a relatively large fabrication tolerance in the OAM EDFA parameters.
\end{abstract}

Keywords: mode-division multiplexing; Erbium-doped fiber amplifier; photonic crystal fibers; orbital angular momentum

\section{Introduction}

Optical communication technology has developed rapidly in the past decades. All kinds of multiplexing and high order modulation technological have greatly increased the capacity of single fibers. The transmission capacity is gradually reaching the limit in that a standard single-mode fiber (SMF) cannot carry more than about 100 Tbit $\cdot \mathrm{s}^{-1}$ of data in the $\mathrm{C}+\mathrm{L}$ band [1]. To meet the stupendously increasing demands for transmission capacity, mode-division multiplexing (MDM), which is one of space-division multiplexing (SDM), has been proposed. MDM utilizing the orthogonality among different orbital angular momentum (OAM) states as the multiplex method has exhibited promising prospects in recent years. An OAM beam is characterized by a helical phase front exp (il $\varphi$ ) (in polar coordinates, 1 is the topological charge, and $\varphi$ is the azimuthal angle), which is an optical vortex beam [2-4]. Theoretically, 1 can be any integer value (that is, OAM has an infinite number of orthogonal eigenstates), which means that OAM has great potential to increase the transmission 
capacity in a MDM system [5]. For communications using OAM beams, there are two key problems to be solved. One is the optical vortex generation, which is the most fundamental technique to implement OAM multiplexing. The optical vortex can be generated by a variety of schemes, such as spatial light modulator [6] and modified interference of different modes [7]. A liquid-crystal spatial light modulator is another promising scheme for generating OAM modes, which exhibits some good properties including simplicity, high efficiency, and reconfiguration [8-10]. Another is the optical vortex beam transmission, which needs the fiber supporting OAM modes. In current research, the ring fiber was designed mostly to transmit OAM modes [11-15]. The circular photonic crystal fiber (C-PCF) was also proposed as a potential OAM fiber structure [16-19]. One fiber can transmit several dozens of mutually orthogonal modes. To date, terabit data transmission based on an OAM fiber has been demonstrated [20]. The transmission distance of the OAM mode in fibers has already reached $50 \mathrm{~km}$ [21]. However, many techniques are still needed for handling the implementation of long-haul MDM systems based on OAM modes. OAM carrier signal amplification is one of the critical techniques.

The Erbium-doped fiber amplifier (EDFA) not only allows the light signal to be amplified online directly but also possesses the same range between amplification wavelength and the low loss wavelength of optical fibers, which is suitable for MDM systems based on OAM modes. However, unlike the single-mode fiber amplifier, there are tens (even dozens) of modes in the OAM fiber amplifier. The transverse distribution of each mode is different. The biggest difference of these mode gains was defined as differential mode gain (DMG) [22]. DMG should be low enough to ensure approximately equal amplification of the multimode in the fiber.

In recent studies, most designs of OAM-EDFA are based on the ordinary circular air-core fiber with a one-layer doped region [23-26]. These OAM-EDFAs perform well in the modal gains but the DMG still needs to be improved. A new design of a two-layer Erbium-doped fiber amplifier transmitting OAM modes was introduced in reference [27] and provides a feasible way to deal with the DMG. They reduce DMG by adjusting the concentrations in the two doped regions. In addition, a one-layer doped region OAM fiber amplifier based on the C-PCF has also been presented by our research team. The amplifier can provide DMG lower than $0.2 \mathrm{~dB}$, which is lower than that of the ordinary circular air-core fiber amplifier [28]. Naturally, the C-PCF structure combined with a two-layer doped region would be a more optimal design for OAM-EDFA. Compared with previous work, this OAM-EDFA can provide better performance in both modal gain and DMG. In addition, our design exhibits a higher tolerance for application.

In this paper, we propose and design a new OAM-EDFA with two-layer Erbium doped based on the C-PCF. The intensity distribution of OAM modes is studied to adjust the doped regions reasonably. The widths and the doped concentrations of the two layers are considered. The performance of the OAM-EDFA with different parameters is analyzed to get an optimal design. All 14 OAM modes are amplified as equally as possible to achieve long-haul transmission.

\section{Theory}

Erbium ion has a unique three-level system, as shown in Figure 1. It includes a ground state, metastable state, and excited state, which is suitable to realize the population inversion between the metastable state and ground state to amplify a signal light whose wavelength is around the 1550 nm window (C-band). We used the Giles and Desurvire model to carry out the analyses upon the population conversion, which is widely used in simulations for fiber amplifiers [29-33]. Owing to the lifetime of the Erbium ion in the metastable state, which is much larger than that in the excited state, the particles of the excited state can be ignored; therefore, the model is considered as a two-level system. Then, we can describe the changing rate of the Erbium ion concentration in the metastable state by the following equations:

$$
\frac{d n_{2}(r, \varphi, z)}{d t}=\sum_{k} \frac{P_{k} i_{k} \sigma_{a k}}{h v_{k}} n_{1}(r, \varphi, z)-\sum_{k} \frac{P_{k} i_{k} \sigma_{e k}}{h v_{k}} n_{2}(r, \varphi, z)-\frac{n_{2}(r, \varphi, z)}{\tau}
$$




$$
n_{t}(r, \varphi, z)=n_{1}(r, \varphi, z)+n_{2}(r, \varphi, z)
$$

where $n_{1}$ and $n_{2}$ are the Erbium ion concentrations of the ground state and the metastable state, respectively; $P_{k}$ is the power of light; $k=s, p, a$ corresponds to the light power of the signal and pump as well as the amplifier spontaneous emission (ASE) noise, respectively; $i_{k}$ is the normalized light intensity; $\sigma_{a k}$ and $\sigma_{e k}$ are the absorption and emission cross-sections (which are the attributes of $\mathrm{Er}^{3+}$ ) respectively; $h$ is Planck constant; and $\tau$ is the lifetime of the metastable state. Equation (2) denotes the particle conservation in the two-level system, where $n_{t}$ is the total Erbium ion concentration. When the EDFA is in a stable state, the number of particles in the metastable state remains unchanged, and Equation (1) is equal to zero. Then, the particle inversion can be given by:

$$
n_{2}(r, \varphi, z)=n_{t} \frac{\sum_{k} \frac{\tau P_{k} i_{k} \sigma_{a k}}{h v_{k}}}{1+\sum_{k} \frac{\tau P_{k} i_{k}\left(\sigma_{a k}+\sigma_{e k}\right)}{h v_{k}}}
$$

The transmission equation in the optical fiber can be expressed by the following propagation equation:

$$
\begin{aligned}
& \frac{d P_{k}}{d z}=\sigma_{e k}\left(P_{k}(z)+m h v_{k} \Delta v_{k}\right) \int_{0}^{2 \pi} \int_{0}^{\infty} i_{k}(r, \Phi) n_{2}(r, \Phi, z) r d r d \Phi \\
& -\sigma_{a k} P_{k}(z) \int_{0}^{2 \pi} \int_{0}^{\infty} i_{k}(r, \Phi) n_{1}(r, \Phi, z) r d r d \Phi
\end{aligned}
$$

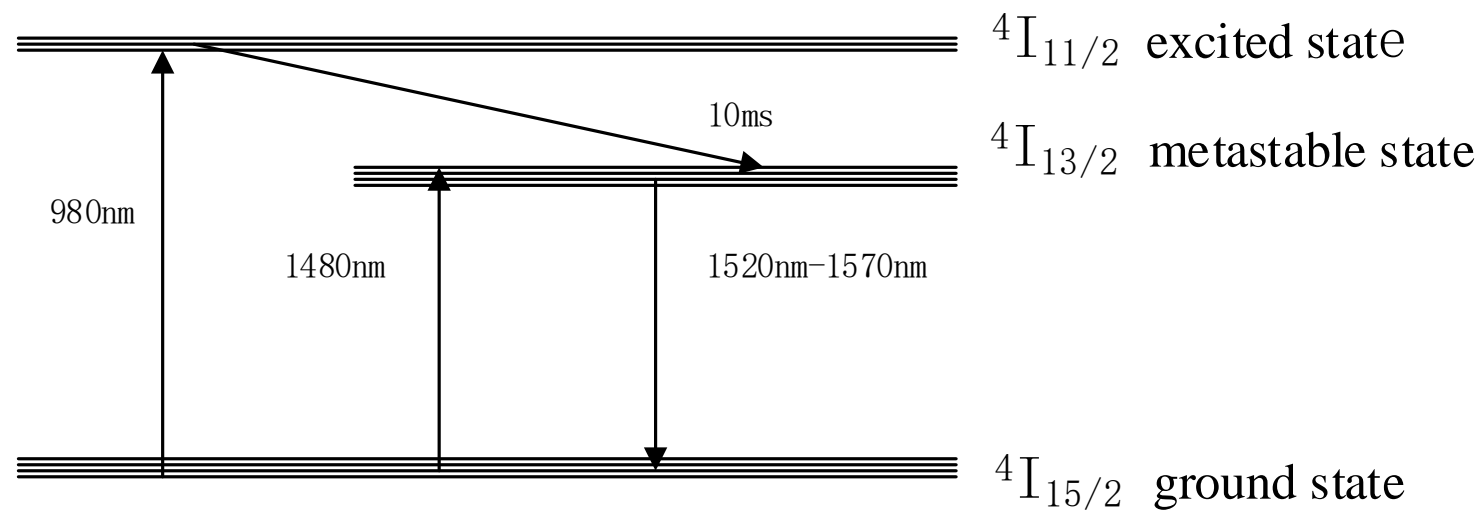

Figure 1. Three-level diagram of Erbium ion.

In the EDFA, the amplification occurs in the process of energy exchange between the pump light and Erbium ions. Therefore, the overlap of the pump light and the doped region determines mainly the performance of EDFA. Therefore, we define the overlap factor as the dimensionless integral overlap between the normalized optical intensity distribution and the normalized Erbium ion distribution:

$$
\Gamma_{k}=\frac{\int_{0}^{2 \pi} \int_{a}^{b} i_{k}(r, \varphi) n_{2}(r, \varphi, z) r d r d \varphi}{\overline{n_{2}}}
$$

where $a$ and $b$ are the inner and outer radius of the fiber doped region. To obtain efficient amplification, it is necessary to make the signal light and the doped area perfectly overlapped, and the signal light and pump light matched greatly. Thus, a correction factor $\eta$ is presented to evaluate the overlap between the signal light and the pump light [28]:

$$
\eta=\frac{\left|\iint E_{s}^{*} \cdot E_{p} d x d y\right|^{2}}{\left|\iint E_{s} \cdot E_{s}^{*} d x d y\right|\left|\iint E_{p} \cdot E_{p}^{*} d x d y\right|}
$$


where $E_{\mathrm{s}}$ and $E_{\mathrm{p}}$ is the signal and pump electric field, respectively. For an accurate description, the overlap factor is modified as $\Gamma_{k}{ }^{\prime}=\Gamma_{k} \times \eta$. The noise figure is another property of EDFA and can be calculated by:

$$
F_{n}=2 n_{s p}(G-1) / G
$$

where $n_{s p}$ and $G$ represent the spontaneous emission factor and the mode gain, respectively. The Equations (1)-(7) are combined to analyze the performance of the amplifier based on the OAM fiber.

\section{Modeling of the OAM-EDFA}

The light intensity distributions of OAM modes transmitted in fiber are ring-shaped. Therefore, the circular fiber structure, such as C-PCF, is a good choice for an OAM fiber. The OAM modes are formed by linearly combining orthogonal even and odd vector modes with a $\pi / 2$ phase shift by the following equations:

$$
\left\{\begin{array}{l}
O A M_{ \pm l, m}^{ \pm}=H E_{l+1, m}^{\text {even }} \pm j H E_{i+1, m}^{\text {odd }} \\
O A M_{ \pm l, m}^{\mp}=E H_{l-1, m}^{\text {even }} \pm j E H_{i-1, m}^{\text {odd }}
\end{array}\right\}(l>1)
$$

where $l$ is called the topological charge representing the number of the azimuthal period, and $\mathrm{m}$ is the radial order giving the number of concentric rings. The superscript " \pm " denotes the polarization state of the OAM mode. OAM modes combined of eigenmode HE possess a circular polarization in the same direction as the OAM rotation, while OAM modes formed of eigenmode EH exhibit a circular polarization in the opposite direction as the OAM rotation [12]. For a given topological charge $(l>1)$ and radial order, four OAM modes form an OAM family. The $\mathrm{OAM}_{1,1}$ family composed of even and odd mode of $\mathrm{HE}_{2,1}$ have two OAM modes because the $\mathrm{OAM}_{1,1}$ composed by $\mathrm{TE}_{0, \mathrm{~m}}$ and $\mathrm{TM}_{0, \mathrm{~m}}$ mode is unstable [34].

The C-PCF fiber structure without the two doped regions in Figure 2a was proposed by our group [17]. It supports the 14 OAM mode transmission and exhibits some good features, such as wide bandwidth, flat dispersion, and low confinement loss. The C-PCF is formed by a large air-hole located at the fiber center, solid circular ring region, and four rings of air-hole arrays as the photonic crystal cladding. The substrate material is pure silica with a refractive index of $1.444($ at $1.55 \mu \mathrm{m})$, and the air region contributes to the refractive index of 1 . Thus, the refractive index of the cladding constituted by the period photonic crystal structure is determined by the weight of the two materials, which can be changed by tailoring the air-filling fraction of the photonic crystal cladding [35]. Between the central air-hole and the cladding air-hole arrays is a ring-shaped area, which acts as the high index fiber ring-core to confine the OAM modes well within it. If we arrange the two-layer Erbium-doped regions as shown in Figure 2a, it would be a good OAM-EDFA structure for both transmission and amplification. The spatial lattice positions on the $x-y$ plane are given by:

$$
x=\Lambda N \cos \left(\frac{2 n \pi}{6 N}\right), y=\Lambda N \sin \left(\frac{2 n \pi}{6 N}\right), n=1-6 N
$$

where $\Lambda$ and $N$ are the lattice constant and number of concentric lattice periods, respectively; $r$ denotes the inner radius of the ring-shaped area with a high index (that is the radius of a large air-hole in the fiber center), and $d_{2}$ to $d_{5}$ are the diameters of the cladding air holes, $\varphi$ is the azimuthal angle. We set the lattice constant of $\Lambda=2 \mu \mathrm{m}$, the diameter of a large air hole of $d_{0}=2.4 \mu \mathrm{m}$, and parameters $d_{n} / \Lambda=0.8, d_{2}=d_{3}=d_{4}=d_{5}=1.6 \mu \mathrm{m}$. 


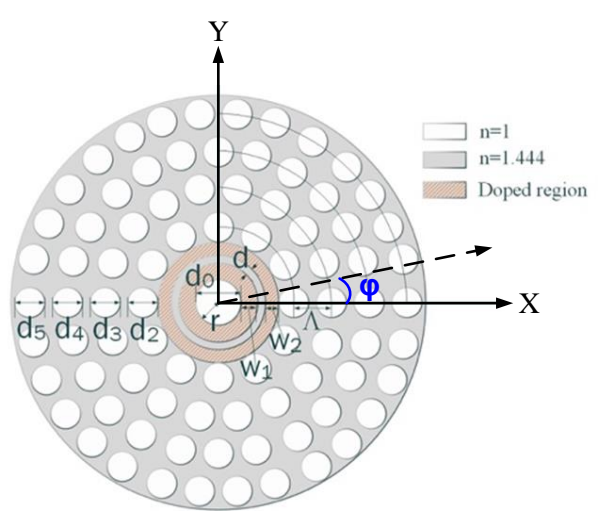

(a)

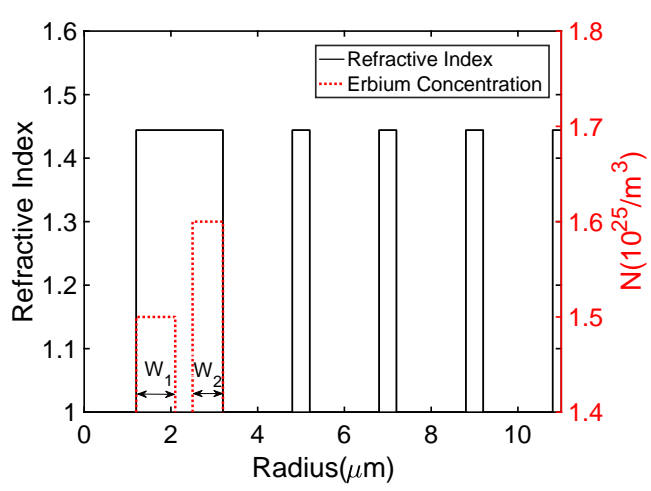

(b)

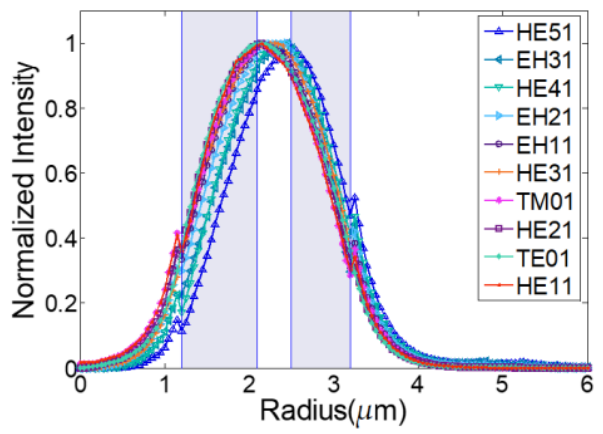

(c)

Figure 2. (a) Schematic of a cross-section and the Erbium-doped region of the circular photonic crystal fiber (C-PCF); (b) the refractive index and Erbium concentration profile of the fiber; (c) normalized intensity profile of the modes in the C-PCF.

The schematic of the two doping regions of EDFA is shown in Figure 2a covered with a red grid. The refractive index and Erbium concentration profile of the fiber is shown in Figure $2 b$ and the two doping regions correspond to the two grey areas in Figure 2c. The normalized intensity distribution of the 14 OAM modes is shown in Figure 2c. We can see that the low order OAM modes tend to distribute in the inner area of the high index region, while the high order OAM modes fall in the outer area. According to Equation (5), the amplification of different OAM modes is determined by the overlapping region between the doped region and the mode field distributions, which will cause different DMGs.

To reduce this difference, we arranged two-layer doped Erbium regions at the inner and outer sides of the high index region. The inner doped region overlaps more with the low order OAM modes, while the outer doped region overlaps more with the high order OAM modes. Therefore, this two-layer Erbium-doped arrangement balances the amplification difference between the lower and higher order OAM modes, and hence minimizes the DMG. Then, the doped widths and Erbium ion concentrations in the two regions should be optimized to obtain the optimal performance.

In the one-layer structure, the design can only be adjusted at the boundary of the doped region where the difference among the intensity of modes is the biggest, which means that the tolerance for application is low. Little doped boundary unconformity will cause a big difference for the performance. However, in the two-layer structure, the design is adjusted where intensities of different modes are very close. The tolerance of our design will be much higher.

\section{Analysis and Optimization}

To design an OAM-EDFA with good features, we need to study the influence of the parameters on the performance of the amplifier. The equilibrium amplification of different OAM modes and the conversion efficiency should be considered to realize low DMG and high gain. Figure 2a shows the structural parameters of the proposed OAM-EDFA, where $w_{1}$ and $w_{2}$ are the widths of the inner 
and outer ring doped region, respectively, which defines the performance of the amplifier. $d$ denotes the spacing of the two-layer doped region, which provides the total effective amplifier area of the EDFA, and, furthermore, determines the conversion efficiency. Next, we investigate the selection of the parameters. First, $w_{1}$ and $w_{2}$ are set as equal, and $d$ is swept from $0.8 \mu \mathrm{m}$ to $0.2 \mu \mathrm{m}$ with a step of $0.2 \mu \mathrm{m}$. Figure 3 shows the gains of different order modes versus $d$ at $1550 \mathrm{~nm}$ wavelength. When $d$ is equal to $0.4 \mu \mathrm{m}$, the gains of different order modes are almost identical, so the DMG is the lowest.

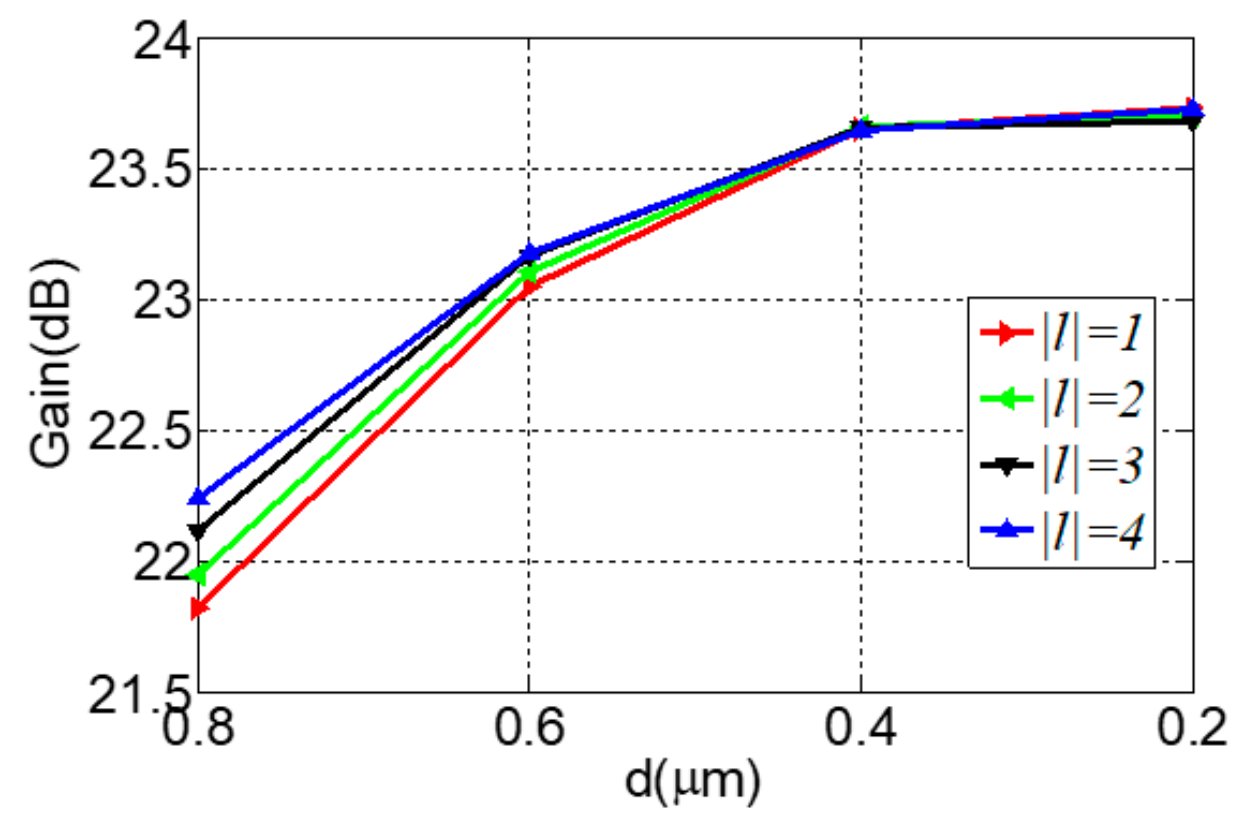

Figure 3. Gain as a function of spacing $d$ at $1550 \mathrm{~nm}$.

Figure $2 \mathrm{c}$ shows that the overlap between the field distribution of the lower order modes and the doped region is larger than that of higher order modes when $w_{1}$ is increased. Thus, the gain of lower order modes will increase faster than that of higher order modes. On the contrary, when $w_{2}$ is increased, the increases in the gain of lower order modes will be slower than that of higher order modes. We can balance the amplification of different modes by increasing $w_{1}$ and decreasing $w_{2}$; accordingly, the DMG can be reduced. The final parameters are tailored to $w_{1}=0.9 \mu \mathrm{m}$ and $w_{2}=0.7 \mu \mathrm{m}$ while $d=0.4 \mu \mathrm{m}$ through further optimization, where the $w_{1}$ is nearly at the point of the intersection of different modes in Figure 2c.

Besides the widths of the doped regions, the doping concentrations and the length of the EDFA also affect the performance. The relationship between the two concentrations of the doped regions and DMG (Erbium-doped profile is assumed uniform in each area) is shown in Figure 4a. The concentration in the circle can be set with an acceptable DMG. However, when the doped concentration is higher than the value of $1.8 \times 10^{25} \mathrm{~m}^{-3}$, the noise figure is higher than $4 \mathrm{~dB}$, as shown in Figure $4 \mathrm{~b}$. Considering to balance the DMG and noise figure, $N_{1}$ is set at $1.5 \times 10^{25} \mathrm{~m}^{-3}$ and $N_{2}$ is set at $1.6 \times 10^{25} \mathrm{~m}^{-3}$ as shown in Figure $2 b$, providing a great result for both low DMG and noise. The length of EDFA is swept from $0 \mathrm{~m}$ to $15 \mathrm{~m}$ as shown in Figure $4 \mathrm{c}$. The mode gains increase rapidly and then gradually saturates with the increase in fiber length, while the noise figure (NF) increases slowly and then rises rapidly. To compromise the mode gains and the noise figure, the length is set to $7 \mathrm{~m}$. 


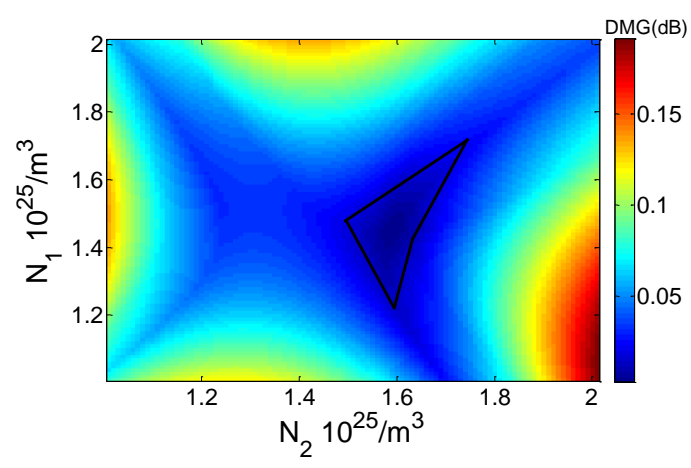

(a)

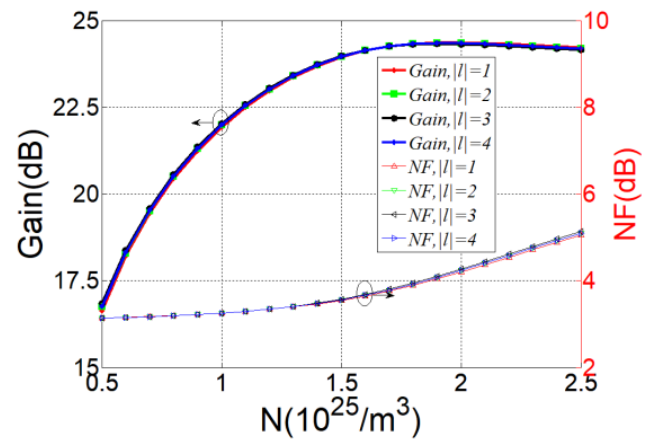

(b)

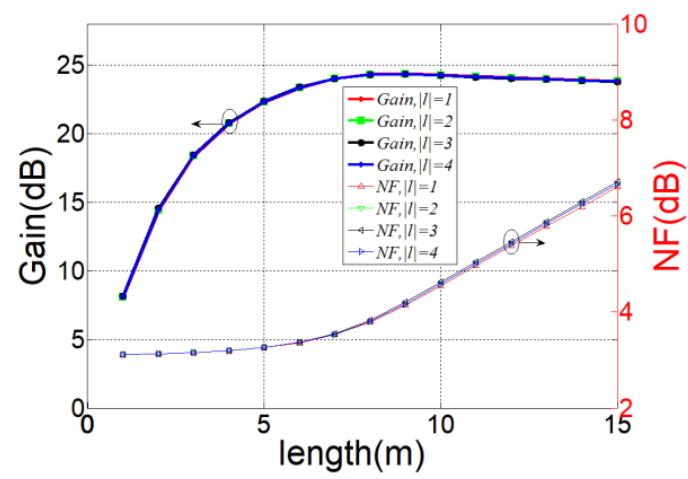

(c)

Figure 4. (a) Differential mode gain (DMG) as a function of the doping concentration $N_{1}$ and $N_{2}$, (b) Gain and noise figure (NF) as a function of the doping concentration N; (c) Gain and NF versus fiber length.

When the parameters of the structure are settled, the mode gains and DMG as functions of the pump power and signal power are shown in Figure 5. The signal wavelength and the pump wavelength are set to $1550 \mathrm{~nm}$ and $980 \mathrm{~nm}$, respectively. The pump power is swept from $50 \mathrm{~mW}$ to $300 \mathrm{~mW}$. The gain first increases rapidly and then gradually becomes saturated, and the DMG changes similarly, as shown in Figure 5a. To get a relatively high gain and low DMG, the pump power is chosen as $150 \mathrm{~mW}$ by a trade-off method where the gain is close to saturation and the DMG is low enough. Figure $5 b$ shows the gain and DMG as the function of input signal power. Considering the trade-off between gain and DMG, we can take the value of the input signal power to be $-15 \mathrm{dBm}$, which provides the gain is larger than $20 \mathrm{~dB}$, and DMG is less than $0.05 \mathrm{~dB}$.

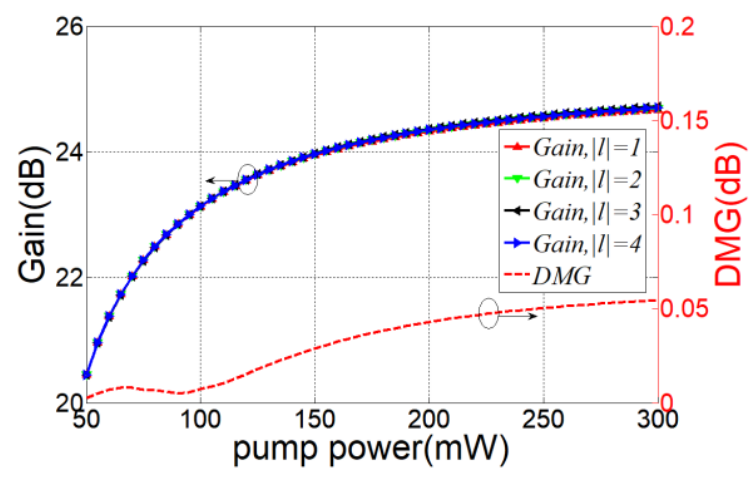

(a)

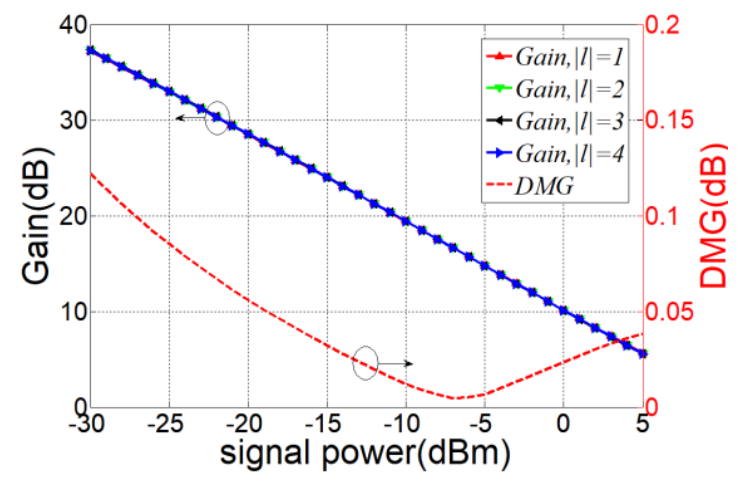

(b)

Figure 5. (a) Gain and DMG versus pump power; (b) Gain and DMG versus signal power. 
Figure 6 exhibits the performance of the OAM-EDFA with optimal parameters. The parameters within the doped region are set to $w_{1}=0.9 \mu \mathrm{m}, w_{2}=0.7 \mu \mathrm{m}, d=0.4 \mu \mathrm{m}, N_{1}=1.5 \times 10^{25} \mathrm{~m}^{-3}$, and $N_{2}=$ $1.6 \times 10^{25} \mathrm{~m}^{-3}$. The length of OAM-EDFA is selected as $7 \mathrm{~m}$. The pump power and signal power are set to $150 \mathrm{~mW}$ and $-15 \mathrm{dBm}$, respectively. The DMG of 14 OAM modes is lower than $0.1 \mathrm{~dB}$, and the noise figures are below $4 \mathrm{~dB}$ over the whole C-band, as shown in Figure 6a. As well, over $23 \mathrm{~dB}$ gain across the C-band is obtained for the OAM-EDFA, as shown in Figure 6b, which can meet the need of MDM systems well.

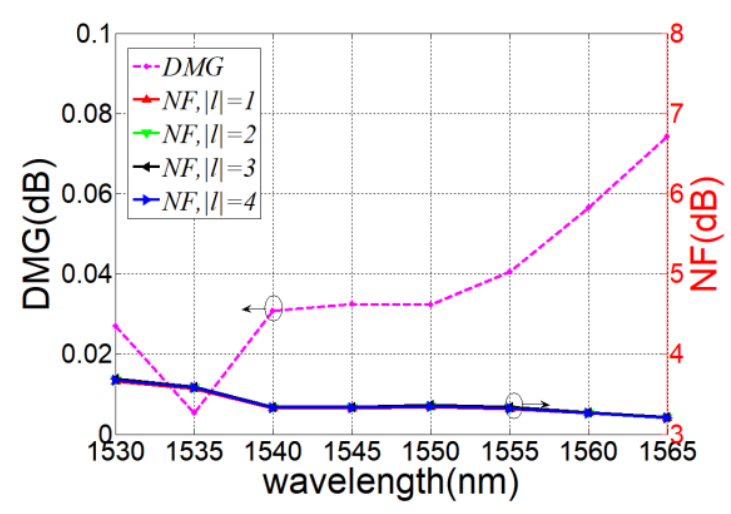

(a)

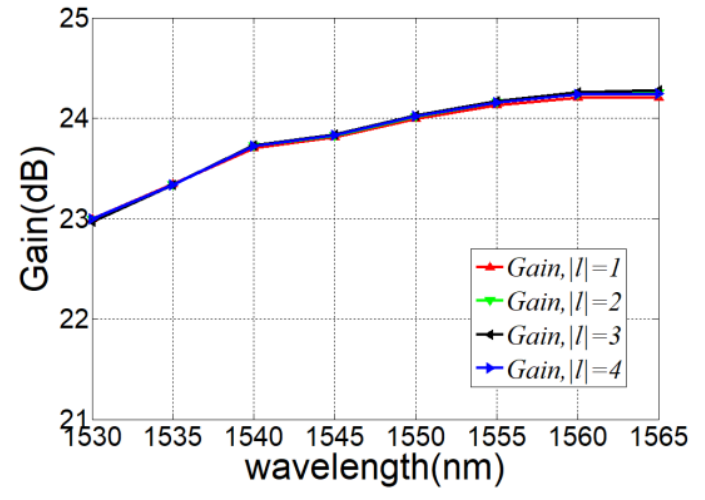

(b)

Figure 6. (a) DMG and NF versus wavelength; (b) Gain versus wavelength.

In Figure 4a, we can see that the DMG would be acceptable when the doping concentrations are in the marked area, which means a large range for concentration tolerance. To obtain a balance between avoiding the radially higher order modes and achieving a good quality of OAM modes, a value of $\mathrm{d} 0$ can be selected from $2.2 \mu \mathrm{m}$ to $3.6 \mu \mathrm{m}$, whose large range indicates a large fabrication tolerance [17]. Besides the concentration and the diameter of a large air-hole, the doped width tolerance should also be considered. Owing to the two-layer structure, OAM-EDFA can equalize the gain of the high order OAM modes and the lower order OAM modes. Therefore, the width between $w_{1}$ and $w_{2}$ can be tolerated within a larger range. At present, the fabrication of PCF and the doping process of the ion have been matured. A fiber similar to the C-PCF structure has been drawn and experimented on [36-38]. Therefore, it is feasible to manufacture the OAM-EDFA based on C-PCF.

\section{Conclusions}

We have presented a new design of OAM-EDFA with a two-layer doped profile to stably maintain 18 eigenmodes (14 OAM modes) for the MDM system. The amplifier is based on the C-PCF supporting OAM modes and adopts the core-pumping scheme. Parameters that affect the amplifier performance, such as the width of the two doped regions, the doping concentrations, the length of the amplifier fiber, and the pump power and signal power, are optimized by a trade-off scheme. The fabrication tolerance of the EDFA is also discussed, and the results show a relatively large tolerance. The two-layer doped region can balance the amplification performance of different modes to minimize the DMG of 14 OAM modes below $0.08 \mathrm{~dB}$. The optimal OAM-EDFA designed will theoretically achieve a gain over $23 \mathrm{~dB}$ and a noise figure less than $4 \mathrm{~dB}$ for all 14 OAM modes across the full C-band.

Author Contributions: H.Z. and L.X. conceived and designed the manuscript, H.Z. wrote the manuscript, and L.X. modified the manuscript and confirmed the final version to be submitted; D.H. and H.L. calculated and analyzed the data; X.Z., Z.Z., and W.Z. investigated the literature and presented formal analyses.

Funding: This research was funded partly by the National Natural Science Foundation of China (NSFC), grant number 61571057, 61527820, 61575082.

Conflicts of Interest: The authors declare no conflict of interest. 


\section{References}

1. Richardson, D.J.; Fini, J.M.; Nelson, L.E. Space-division multiplexing in optical fibres. Nat. Photonics 2013, 7, 354-362. [CrossRef]

2. Allen, L.; Beijersbergen, M.W.; Spreeuw, R.; Woerdman, J. Orbital angular momentum of light and the transformation of Laguerre-Gaussian laser modes. Phys. Rev. A 1992, 45, 8185-8189. [CrossRef] [PubMed]

3. Ramachandran, S.; Kristensen, P. Optical vortices in fiber. Nanophotonics 2013, 2, 455-474. [CrossRef]

4. Brunet, C.; Rusch, L.A. Optical fibers for the transmission of orbital angular momentum modes. Opt. Fiber Technol. 2016, 31, 172-177. [CrossRef]

5. Yao, A.M.; Padgett, M.J. Orbital angular momentum: Origins, behavior and applications. Adv. Opt. Photonics 2011, 3, 161-204. [CrossRef]

6. Chen, H.; Hao, J.; Zhang, B.; Xu, J.; Ding, J.; Wang, H. Generation of vector beam with space-variant distribution of both polarization and phase. Opt. Lett. 2011, 36, 3179-3181. [CrossRef] [PubMed]

7. Robert, D.N.; Mark, E.S.; Juliet, T.G. Continuously tunable orbital angular momentum generation using a polarization-maintaining fiber. Opt. Lett. 2016, 41, 3213-3216.

8. José, F.A.; Urruchi, V.; Braulio, G.; José, M.S. Generation of optical vortices by an ideal liquid crystal spiral phase plate. IEEE Electron Device Lett. 2014, 35, 856-858. [CrossRef]

9. Andrey, S.O.; Rickenstorff-Parrao, C.; Arrizón, V. Generation of the "perfect" optical vortex using a liquid-crystal spatial light modulator. Opt. Lett. 2013, 38, 534-536.

10. Kotova, S.P.; Mayorova, A.M.; Samagin, S.A. Formation of ring-shaped light fields with orbital angular momentum using a modal type liquid crystal spatial modulator. J. Opt. 2018, 20, 055604. [CrossRef]

11. Yan, Y.; Yue, Y.; Huang, H.; Yang, J.; Chitgarha, M.R.; Ahmed, N.; Tur, M.; Dolinar, S.J.; Willner, A.E. Efficient generation and multiplexing of optical orbital angular momentum modes in a ring fiber by using multiple coherent inputs. Opt. Lett. 2012, 37, 3645-3647. [CrossRef] [PubMed]

12. Li, S.; Wang, J. Multi-orbital-angular-momentum multi-ring fiber for high-density space-division multiplexing. IEEE Photonics J. 2013, 5. [CrossRef]

13. Yue, Y.; Yan, Y.; Ahmed, N.; Yang, J.; Zhang, L.; Ren, Y.; Huang, H.; Birnbaum, K.M.; Erkmen, B.I.; Dolinar, S.; et al. Mode properties and propagation effects of optical orbital angular momentum (OAM) modes in a ring fiber. IEEE Photonics J. 2012, 4, 535-543.

14. Brunet, C.; Vaity, P.; Messaddeq, Y.; LaRochelle, S.; Rusch, L.A. Design, fabrication and validation of an OAM fiber supporting 36 states. Opt. Express 2014, 22, 26117-26127. [CrossRef] [PubMed]

15. Wang, L.; Nejad, R.M.; Corsi, A.; Lin, J.; Messaddeq, Y.; LaRochelle, S.; Rusch, L.A. Linearly polarized vector modes: Enabling MIMO-free mode-division multiplexing. Opt. Express 2017, 25, 11736-11749. [CrossRef] [PubMed]

16. Zhang, H.; Zhang, X.; Li, H.; Deng, Y.; Xi, L.; Tang, X.; Zhang, W. The orbital angular momentum modes supporting fibers based on the photonic crystal fiber structure. Crystals 2017, 7, 286. [CrossRef]

17. Zhang, H.; Zhang, W.; Xi, L.; Tang, X.; Zhang, X.; Zhang, X. A new type circular photonic crystal fiber for orbital angular momentum mode transmission. IEEE Photonics Technol. Lett. 2016, 28, 1426-1429. [CrossRef]

18. Zhang, H.; Zhang, W.; Xi, L.; Tang, X.; Zhang, X. A New Design of a Circular Photonic Crystal Fiber Supporting 42 OAM Modes. In Proceedings of the Australian Conference on Optical Fibre Technology, Sydney, Australia, 5-8 September 2016; pp. 1-2.

19. Zhang, H.; Zhang, X.; Li, H.; Deng, Y.; Zhang, X.; Xi, L.; Tang, X.; Zhang, W. A design strategy of the circular photonic crystal fiber supporting good quality orbital angular momentum mode transmission. Opt. Commun. 2017, 397, 59-66. [CrossRef]

20. Bozinovic, N.; Yue, Y.; Ren, Y.; Tur, M.; Kristensen, P.; Huang, H.; Willner, A.E.; Ramachandran, S. Terabit scale orbital angular momentum mode division multiplexing in fibers. Science 2013, 340, 1545-1548. [CrossRef] [PubMed]

21. Wang, A.; Zhu, L.; Chen, S.; Du, C.; Mo, Q.; Wang, J. Characterization of LDPC-coded orbital angular momentum modes transmission and multiplexing over a $50 \mathrm{~km}$ fiber. Opt. Express 2016, 24, 11716-11726. [CrossRef] [PubMed]

22. Qian, X.; Boucouvalas, A.C. Propagation Characteristics of single-mode optical fibers with arbitrary complex index profiles. IEEE J. Quantum Elect. 2006, 40, 771-777. [CrossRef] 
23. Kang, Q.; Gregg, P.; Jung, Y.; Lim, E.L.; Alam, S.; Ramachandran, S.; Richardson, D.J. Amplification of 12 OAM Modes in an air-core erbium doped fiber. Opt. Express 2015, 23, 28341-28348. [CrossRef] [PubMed]

24. Kang, Q.; Lim, E.L.; Jung, Y.; Poletti, F.; Alam, S.; Richardson, D.J. Design of Four-Mode Erbium Doped Fiber Amplifier with Low Differential Modal Gain for Modal Division Multiplexed Transmissions. In Proceedings of the Optical Fiber Communication Conference and Exposition and the National Fiber Optic Engineers Conference IEEE, Anaheim, CA, USA, 17-21 March 2013; pp. 1-3.

25. Kang, Q.; Lim, E.L.; Poletti, F.; Jung, Y.; Baskiotis, C.; Alam, S.; Richardson, D.J. Minimizing differential modal gain in cladding-pumped EDFAs supporting four and six mode groups. Opt. Express 2014, 22, 21499-21507. [CrossRef] [PubMed]

26. Jung, Y.; Kang, Q.; Sidharthan, R.; Ho, D.; Yoo, S.; Gregg, P.; Ramachandran, S.; Alam, S.; Richardson, D.J. Optical orbital angular momentum amplifier based on an air-hole erbium-doped fiber. J. Lightwave Technol. 2017, 35, 430-436. [CrossRef]

27. Ma, J.; Xia, F.; Li, S.; Wang, J. Design of Orbital Angular Momentum (OAM) Erbium Doped Fiber Amplifier with Low Differential Modal Gain. In Proceedings of the Optical Fiber Communication Conference and Exposition/National Fiber Optic Engineers Conference IEEE, Los Angeles, CA, USA, 22-26 March 2015; pp. 1-3.

28. Deng, Y.; Zhang, H.; Li, H.; Tang, X.; Zhang, X.; Xi, L.; Zhang, W.; Zhang, X. Erbium-doped amplification in circular photonic crystal fiber supporting orbital angular momentum modes. Appl. Opt. 2017, 56, 1748-1752. [CrossRef] [PubMed]

29. Giles, C.R.; Desurvire, E. Modeling erbium-doped fiber amplifiers. J. Lightwave Technol. 1991, 9, $271-283$. [CrossRef]

30. Bigot, L.; Le Cocq, G.; Quiquempois, Y. Few-mode erbium-doped fiber amplifiers: A review. J. Lightwave Technol. 2015, 33, 588-596. [CrossRef]

31. Varshney, S.K.; Saitoh, K.; Koshiba, M.; Pal, B.P.; Sinha, R.K. Design of S-band erbium-doped concentric dual-core photonic crystal fiber amplifiers with ASE suppression. J. Lightwave Technol. 2009, 27, 1725-1733. [CrossRef]

32. Le Cocq, G.; Quiquempois, Y.; Le Rouge, A.; Bouwmans, G.; El Hamzaoui, H.; Delplace, K.; Bouazaoui, M.; Bigot, L. Few mode $\mathrm{Er}^{3+}$-doped fiber with micro-structured core for mode division multiplexing in the C-band. Opt. Express 2013, 21, 31646-31659. [CrossRef] [PubMed]

33. Saitoh, S.; Saitoh, K.; Kashiwagi, M.; Matsuo, S.; Dong, L. Design optimization of large-mode-area all-solid photonic bandgap fibers for high-power laser applications. J. Lightwave Technol. 2014, 32, 440-449. [CrossRef]

34. Zhou, G.; Zhou, G.; Chen, C.; Xu, M.; Xia, C.; Hou, Z. Design and analysis of a microstructure ring fiber for orbital angular momentum transmission. IEEE Photonics J. 2017, 8, 1-12. [CrossRef]

35. Li, H.; Zhang, H.; Zhang, X.; Zhang, Z.; Xi, L.; Tang, X.; Zhang, W.; Zhang, X. Design tool for circular photonic crystal fibers supporting orbital angular momentum modes. Appl. Opt. 2018, 57, 2474-2481. [CrossRef] [PubMed]

36. Tandje, A.; Yammine, J.; Bouwmans, G.; Dossou, M.; Vianou, A.; Andresen, E.R.; Bigot, L. Design and Fabrication of a Ring-Core Photonic Crystal Fiber for Low-Crosstalk Propagation of OAM Modes. In Proceedings of the European Conference on Optical Communication IEEE, Rome, Italy, 23-25 September 2018; pp. 1-3.

37. Chen, Y.; Liu, Z.; Sandoghchi, S.R.; Jasion, G.T.; Bradley, T.D.; Fokoua, E.N.; Hayes, J.R.; Wheeler, N.V.; Gray, D.R.; Mangan, B.J.; et al. Multi-kilometer long, longitudinally uniform hollow core photonic bandgap fibers for broadband low latency data transmission. J. Lightwave Technol. 2016, 34, 104-113. [CrossRef]

38. Foroni, M.; Passaro, D.; Poli, F.; Cucinotta, A.; Selleri, S.; Lægsgaard, J.; Bjarklev, A.O. Guiding properties of silica/air hollow-core bragg fibers. J. Lightwave Technol. 2008, 26, 1877-1884. [CrossRef]

(C) 2019 by the authors. Licensee MDPI, Basel, Switzerland. This article is an open access article distributed under the terms and conditions of the Creative Commons Attribution (CC BY) license (http://creativecommons.org/licenses/by/4.0/). 